OPEN ACCESS

Edited by:

Fu-Li Li,

Qingdao Institute of Bioenergy and Bioprocess Technology (CAS),

China

Reviewed by:

Noppol - Leksawasdi,

Chiang Mai University, Thailand Xiangzhao Mao,

Ocean University of China, China Md. Abu Saleh,

University of Rajshahi, Bangladesh

*Correspondence: Xiuwen Wang wangxiuwen0822@163.com Jiayang Qin qinjysdu@163.com

Specialty section This article was submitted to Bioprocess Engineering,

a section of the journal Frontiers in Bioengineering and Biotechnology

Received: 13 February 2020

Accepted: 19 March 2020

Published: 22 April 2020

Citation:

Wang A, Tian W, Cheng L, XU Y,

Wang $X$, Qin J and Yu B (2020) Enhanced $\varepsilon$-Poly-L-Lysine Production by the Synergistic Effect

of $\varepsilon$-Poly-L-Lysine Synthetase

Overexpression and Citrate

in Streptomyces albulus.

Front. Bioeng. Biotechnol. 8:288.

doi: 10.3389/fbioe.2020.00288

\section{Enhanced $\varepsilon$-Poly-L-Lysine Production by the Synergistic Effect of $\varepsilon$-Poly-L-Lysine Synthetase Overexpression and Citrate in Streptomyces albulus}

\author{
Aixia Wang ${ }^{1}$, Wenzhe Tian', Lei Cheng ${ }^{2}$, Youqiang $X u^{2}$, Xiuwen Wang ${ }^{1 *}$, Jiayang Qin ${ }^{1 *}$ \\ and $\mathrm{Bo} \mathrm{Yu}^{3}$
}

\begin{abstract}
${ }^{1}$ College of Pharmacy, Binzhou Medical University, Yantai, China, ${ }^{2}$ Beijing Engineering and Technology Research Center of Food Additives, Beijing Technology and Business University (BTBU), Beijing, China, ${ }^{3}$ CAS Key Laboratory of Microbial Physiological and Metabolic Engineering, Institute of Microbiology, Chinese Academy of Sciences, Beijing, China
\end{abstract}

$\varepsilon$-Poly-L-lysine $(\varepsilon-P L)$ is a natural amino acid polymer produced by microbial fermentation. It has been mainly used as a preservative in the food and cosmetics industries, as a drug carrier in medicines, and as a gene carrier in gene therapy. $\varepsilon-P L$ synthase is the key enzyme responsible for the polymerization of L-lysine to form $\varepsilon$-PL. In this study, the $\varepsilon-P L$ synthase gene was overexpressed in Streptomyces albulus CICC 11022 by using the kasOp* promoter and the ribosome binding site from the capsid protein of phage $\phi C 31$, which resulted in a genetically engineered strain Q-PL2. The titers of $\varepsilon$-PL produced by Q-PL2 were $88.2 \% \pm 8.3 \%$ higher than that produced by the wild strain in shake flask fermentation. With the synergistic effect of $2 \mathrm{~g} / \mathrm{L}$ sodium citrate, the titers of $\varepsilon$-PL produced by Q-PL2 were $211.2 \% \pm 17.4 \%$ higher than that produced by the wild strain. In fed-batch fermentations, $20.1 \pm 1.3 \mathrm{~g} / \mathrm{L}$ of $\varepsilon$-PL was produced by S. albulus Q-PL2 in $72 \mathrm{~h}$ with a productivity of $6.7 \pm 0.4 \mathrm{~g} / \mathrm{L} /$ day, which was $3.2 \pm 0.3$ fold of that produced by the wild strain. These results indicate that $\varepsilon-P L$ synthase is one of the rate-limiting enzymes in $\varepsilon-P L$ synthesis pathway and lays a foundation for further improving the $\varepsilon-P L$ production ability of $S$. albulus by metabolic engineering.

\footnotetext{
Keywords: Streptomyces albulus, $\varepsilon$-PL synthase, overexpression, citrate, synergistic effect
}

\section{INTRODUCTION}

$\varepsilon$-Poly-L-lysine ( $\varepsilon$-PL) is a kind of polymer composed of 25-35 L-lysine residues connected by $\alpha$-amino group and $\varepsilon$-carboxyl group to form an amide bond. It was first separated and purified by Japanese scholars Shima and Sakai in the fermentation broth of Streptomyces (Shima and Sakai, 1977). $\varepsilon$-PL has many excellent properties, including that it is antibacterial, biodegradable, water soluble, thermostable, edible, and non-toxic. Therefore, $\varepsilon$-PL has been used as a preservative in food, cosmetics, health care, and other industries (Xu et al., 2016). In addition, $\varepsilon-\mathrm{PL}$ can also be used as gene carrier, drug carrier, in weight loss and health care products, as a new water absorbent material, biochip, and bioelectronic coating agent (Xu et al., 2016). 
$\varepsilon$-Poly-L-lysine is produced mainly by microbial fermentation. It was proven that L-lysine is the precursor of $\varepsilon$-PL synthesis by using isotope technology (Shima et al., 1983). Kawai et al. (2003) reported the synthesis of $\varepsilon$-PL by using a cell-free system. They found that the synthesis activity was on the cell membrane, and the enzyme activity depended on ATP and was not affected by ribonuclease, kanamycin, or chloramphenicol. These results indicate that $\varepsilon$-PL synthesis is a membrane protein-catalyzed reaction. Five years later, the $\varepsilon$-PL synthase (Pls) was purified from Streptomyces albulus NBRC 14147 (Yamanaka et al., 2008). It was found that the enzyme has a molecular weight of $130 \mathrm{kDa}$ and is an unusual non-ribosomal peptide synthetase with adenylation. In addition, the thiolation domain, without the condensation or thioesterase domain of a traditional peptide synthetase, has six transmembrane domains surrounding three tandem soluble domains, which use free L-lysine polymers (or the monomer in the initial reaction) as a receptor and Pls-bound L-lysine as a donor to continuously catalyze the polymerization of L-lysine, directly producing chains of different lengths. After that, Yamanaka et al. (2011) reported a recombinant Pls expression system that can be used for site-specific mutation analysis. They attempted to express Pls in $S$. albulus using the constitutive promoter $\mathrm{ermE}^{*}$ but were unsuccessful. Therefore, they speculated that the expression of Pls gene $(p l s)$ requires its own promoter, which is likely regulated in secondary metabolism. The pls gene from $S$. albulus was heterologously expressed in S. lividans, and the recombinant strain was capable of synthesizing $\varepsilon$-PL. The own promoter of $p l s$ is still used in their study (Geng et al., 2014). Recently, Xu et al. (2019) found a strain capable of producing short-chain $\varepsilon$-PL, Kitasatospora aureofaciens, and they attempted to heterologously express the plsII gene of this strain in S. albulus. They tested three promoters, namely, the constitutive promoter $\mathrm{ermE}^{*}$, the plsII gene's own promoter from $K$. aureofaciens, and the plsI gene's own promoter from $S$. albulus. The results showed that ermE* $E^{*}$ could not start the expression of plsII gene, whereas the other two promoters could. The final $\varepsilon$-PL concentration obtained by the plsI gene's own promoter from S. albulus was $34.1 \%$ higher than that by the plsII gene's own promoter from $K$. aureofaciens.

The above results indicate that there is no precedent for the successful expression of pls gene using other promoters, except the gene's own promoter. In this study, an engineered strong promoter kasOp* (Wang et al., 2013) and the ribosome binding site (RBS) from the capsid protein of phage $\phi C 31$ (Smith et al., 1999; Bai et al., 2015) were combined to overexpress the pls gene in $S$. albulus, and the ability of the gene-engineered strain to produce $\varepsilon$-PL was also investigated.

\section{MATERIALS AND METHODS}

\section{Strains and Plasmids}

The strains and plasmids used in this study are shown in Table 1. The $\varepsilon$-PL production strain S. albulus CICC 11022 was purchased from the China Industrial Microbial Culture
Collection (CICC). Escherichia coli ET12567/pUZ8002 (Paget et al., 1999) was used as the non-methylating plasmid donor strain for intergeneric conjugation with S. albulus CICC 11022. The E. coli/S. albulus shuttle vector, pSET152, which can integrate specifically into the attB sites on the Streptomyces chromosome via integrase-attp-directed site-specific recombination, was used for $p l s$ gene overexpression.

\section{Strain Culture and Fermentation Conditions}

Escherichia coli was cultured under aerobic condition at $37^{\circ} \mathrm{C}$ using Luria-Bertani (LB) medium, which contained $10 \mathrm{~g} / \mathrm{L}$ tryptone, $5 \mathrm{~g} / \mathrm{L}$ yeast extract, and $10 \mathrm{~g} / \mathrm{L}$ sodium chloride. $S$. albulus was cultured at $30^{\circ} \mathrm{C}$. The medium used to culture the spores of $S$. albulus was MS solid medium, which contains $20 \mathrm{~g} / \mathrm{L}$ of mannitol, $20 \mathrm{~g} / \mathrm{L}$ of soybean powder, and $20 \mathrm{~g} / \mathrm{L}$ of agar powder. M3G medium was used for the seed culture of $S$. albulus, which is composed of $50 \mathrm{~g} / \mathrm{L}$ of glucose, $10 \mathrm{~g} / \mathrm{L}$ of $\left(\mathrm{NH}_{4}\right)_{2} \mathrm{SO}_{4}, 5 \mathrm{~g} / \mathrm{L}$ of yeast extract, $0.5 \mathrm{~g} / \mathrm{L}$ of $\mathrm{MgSO}_{4} \cdot 7 \mathrm{H}_{2} \mathrm{O}, 0.8 \mathrm{~g} / \mathrm{L}$ of $\mathrm{K}_{2} \mathrm{HPO}_{4}, 1.36 \mathrm{~g} / \mathrm{L}$ of $\mathrm{KH}_{2} \mathrm{PO}_{4}, 0.03 \mathrm{~g} / \mathrm{L}$ of $\mathrm{FeSO}_{4} \cdot 7 \mathrm{H}_{2} \mathrm{O}, 0.04 \mathrm{~g} / \mathrm{L}$ of $\mathrm{ZnSO}_{4} \cdot 7 \mathrm{H}_{2} \mathrm{O}$ and has an initial $\mathrm{pH}$ of 6.8. For shake flask fermentations of S. albulus, M3G medium was used as the fermentation medium after appropriate modification according to the experimental requirements. For fed-batch fermentations of S. albulus, $25 \mathrm{~g} / \mathrm{L}$ glucose and $25 \mathrm{~g} / \mathrm{L}$ glycerin were used as the mixed carbon source, $5 \mathrm{~g} / \mathrm{L}$ sodium citrate was added, and the other components were the same as M3G medium without glucose. When required, antibiotics were used at the following concentrations: $50-80 \mu \mathrm{g} / \mathrm{mL}$ apramycin, 25-50 $\mu \mathrm{g} / \mathrm{mL}$ chloramphenicol, 40-50 $\mu \mathrm{g} / \mathrm{mL}$ kanamycin, and $25 \mu \mathrm{g} / \mathrm{mL}$ nalidixic acid.

In the shake flask fermentations of $\varepsilon$-PL, the $S$. albulus strains were streaked onto MS solid medium, and spores were collected after 5-6 days of cultivation at $30^{\circ} \mathrm{C}$. Then, $400-\mu \mathrm{L}$ spore solution was inoculated into $300-\mathrm{mL}$ conical flask containing $50 \mathrm{~mL}$ seed medium, and seed culture was obtained after $48 \mathrm{~h}$ of shaking cultivation at $30^{\circ} \mathrm{C}$ and $220 \mathrm{rpm}$ unless otherwise specified. Then, the seed culture was inoculated into conical flasks containing $50 \mathrm{~mL}$ of fermentation medium at $10 \%$ (volume ratio). After $72 \mathrm{~h}$ of cultivation at $30^{\circ} \mathrm{C}$ and $220 \mathrm{rpm}$, the concentration of $\varepsilon$-PL and other parameters in the fermentation broth were determined.

Fed-batch fermentations of $\varepsilon$-PL by $S$. albulus were conducted in a 2-L bioreactor (NBS, Germany) containing $1 \mathrm{~L}$ of fermentation medium. The preparation of the seed culture was the same as that in shake flask fermentation. The seed culture was inoculated into the fermenter at $10 \%$ volume ratio, and the temperature was controlled at $30^{\circ} \mathrm{C}$. The aeration was $3 \mathrm{vvm}$, and the dissolved oxygen level was controlled at approximately $30 \%$ by adjusting the stirring speed at $200-800 \mathrm{rpm}$. According to Ren et al. (2015), the pH was not controlled in the early stage of fermentation. When the $\mathrm{pH}$ of the fermentation broth dropped to 4.0 , ammonium hydroxide was added to maintain the $\mathrm{pH}$ at 4.0. Feeding medium was added to the fermentation broth when the glucose concentration in the fermentation broth was less than $10 \mathrm{~g} / \mathrm{L}$. The composition of the feeding medium was $250 \mathrm{~g} / \mathrm{L}$ of 
TABLE 1 | Strains and plasmids used in this study.

\begin{tabular}{|c|c|c|}
\hline Strain and plasmid & Relevant genotype ${ }^{a}$ & Source or references \\
\hline \multicolumn{3}{|l|}{ Strains } \\
\hline Streptomyces albulus CICC 11022 & Control strain, transformation host & CICC \\
\hline S. albulus Q-PL2 & Overexpression strain, S. albulus CICC 11022 harboring pSET152-pro-rbs2-pls & This study \\
\hline Escherichia coli ET12567/pUZ8002 & recE, $d c^{-}, d_{a m}^{-}, h s d S, \mathrm{Cm}^{r}, \operatorname{Tet}^{r}, \mathrm{Str}^{\mathrm{r}}, \mathrm{Km}^{\mathrm{r}}$ & Paget et al., 1999 \\
\hline E. coli Top10 & $\begin{array}{l}\mathrm{F}^{-} \text {mcrA } \Delta(\mathrm{mrr}-\mathrm{hsdRMS}-\mathrm{mcrBC}) \text { } 980 \mathrm{lacZ} \Delta \mathrm{M} 15 \Delta \text { lacX74 recA1 araD139 } \Delta \text { (ara-leu)7697 galU } \\
\text { galKrpsL }\left(\mathrm{Str}^{\mathrm{R}}\right) \text { endA1 nupG }\end{array}$ & Invitrogen \\
\hline \multicolumn{3}{|c|}{ 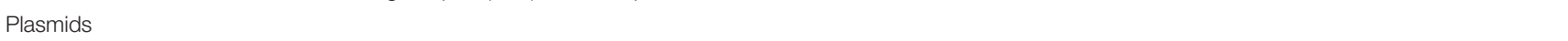 } \\
\hline pSET152 & $5.7 \mathrm{~kb}, \mathrm{Apr}^{\mathrm{r}}$, integrative plasmid, lacZ $\alpha$ oripUC19 ${ }^{\mathrm{ori}} \mathrm{T}^{\mathrm{RP} 4}{ }_{\text {int-attP }}{ }^{31}$ aac(3) $/ \mathrm{V}$ & Bierman et al., 1992 \\
\hline pSET152-pro-rbs2-pls & Apr $^{r}$, pSET152 carrying kasOp* promoter, RBS2, and pls gene & This study \\
\hline
\end{tabular}

${ }^{a} \mathrm{Cm}$, chloramphenicol; Tet, tetracycline; Str, streptomycin; Km, kanamycin; Apr, apramycin.

glucose, $250 \mathrm{~g} / \mathrm{L}$ of glycerin, $100 \mathrm{~g} / \mathrm{L}$ of ammonium sulfate, and $50 \mathrm{~g} / \mathrm{L}$ of sodium citrate. Cell growth, substrate consumption, and $\varepsilon$-PL production were measured every few hours. Three separate experiments were conducted for all the shake flask and fed-batch fermentations.

\section{Molecular Manipulations}

Genomic DNA was extracted using the TIANamp Bacteria DNA Kit (TIANGEN, China). Phanta Super-Fidelity DNA Polymerase was used to amplify the pls gene (Vazyme, China). Plasmid DNA was isolated using the Easypure ${ }^{\circledR}$ Plasmid Miniprep Kit (Transgen, China). The EasyPure ${ }^{\circledR}$ Quick Gel Extraction Kit (Transgen, China) was used for DNA purification. Oligonucleotides were prepared by Invitrogen (Shanghai, China). The Gibson Assembly ${ }^{\circledR}$ Cloning Kit (NEB, England) was used to integrate the vector and the DNA inserts.

\section{Construction of $p / s$ Gene Overexpressing Strain}

The oligonucleotides used to construct $p l s$ gene overexpression plasmids are shown in Supplementary Table S1. The ligation product pro-rbs 2 of the strong promoter kasOp* and the ribosome-binding site (RBS2) from the capsid protein of phage $\phi$ C31 were obtained by annealing oligonucleotides pro1, pro2, and pro4. The primers pls-F2 and pls-R were used to amplify the pls gene from the genome of $S$. albulus CICC 11022. The PCR conditions were as follows: $94^{\circ} \mathrm{C}$ for $30 \mathrm{~s}, 55^{\circ} \mathrm{C}$ for $30 \mathrm{~s}$, and $72^{\circ} \mathrm{C}$ for $2 \mathrm{~min}$, for 30 repeated cycles each. The DNA fragments of pro-rbs 2 and pls gene were mixed as the template to perform overlapping PCR using primers prol and pls-R, and a complete expression element, namely, pro-rbs2-plscontaining the strong promoter $\mathrm{kasOp}^{*}, \mathrm{RBS} 2$, and pls genewas finally obtained. The PCR conditions wer as follows: $94^{\circ} \mathrm{C}$ for $30 \mathrm{~s}, 55^{\circ} \mathrm{C}$ for $30 \mathrm{~s}$, and $72^{\circ} \mathrm{C}$ for $2 \mathrm{~min}$, for 30 repeated cycles each. The expression element pro-rbs2-pls was ligated with XbaI and EcoRI double-digested vector pSET152 by Gibson reaction and transformed into E. coli Top10. The correctly ligated transformants were screened and verified by sequencing. The sequence of pls gene in S. albulus CICC 11022 shows $100 \%$ identity to the pls gene in S. albulus PD-1 (accession no. JF427577). The recombinant overexpression plasmid was named
pSET152-pro-rbs2-pls (Supplementary Figure S1), and the sequence of pro-rbs2-pls is shown in Supplementary Figure S2.

The constructed pls gene overexpression plasmids pSET152pro-rbs2-pls were first transferred into E. coli ET12567/pUZ8002 and then transferred into S. albulus CICC 11022 by intergeneric conjugation, according to a previously reported method (Xu et al., 2015) with some modifications. The specific steps were as follows.

A single colony of E. coli ET12567/pUZ8002 harboring pSET152-pro-rbs2-pls was selected from LB solid medium containing $50 \mu \mathrm{g} / \mathrm{mL}$ kanamycin, $50 \mu \mathrm{g} / \mathrm{mL}$ chloramphenicol, and $50 \mu \mathrm{g} / \mathrm{mL}$ apramycin. The cells were collected at $37^{\circ} \mathrm{C}$ at an $\mathrm{OD}_{600}$ of 0.6 , washed three times with fresh LB medium, and finally resuspended in $200 \mu \mathrm{L}$ LB for later use. Meanwhile, the spores of $S$. albulus CICC 11022 were suspended in $400 \mu \mathrm{L}$ of $2 \times$ YT medium, which contains $16 \mathrm{~g} / \mathrm{L}$ tryptone, $10 \mathrm{~g} / \mathrm{L}$ yeast extract, and $5 \mathrm{~g} / \mathrm{L}$ sodium chloride. After a $10 \mathrm{~min}$ heat shock at $50^{\circ} \mathrm{C}$, the spores were cooled to room temperature, mixed with the prepared donor strain, and cultivated at $30^{\circ} \mathrm{C}$ for $1 \mathrm{~h}$ with shaking at $100 \mathrm{rpm} / \mathrm{min}$. The mixed bacterial solutions were plated onto MS solid medium. After $14 \mathrm{~h}$, the plates were covered with $1 \mathrm{~mL}$ of sterile water containing $80 \mu \mathrm{g} / \mathrm{mL}$ apramycin and $25 \mu \mathrm{g} / \mathrm{mL}$ nalidixic acid. The plates were further incubated at $30^{\circ} \mathrm{C}$ for approximately 2 days, and the ex-conjugants were obtained. The spores were cultured to obtain the genetically engineered strain S. albulus Q-PL2, which harbor pSET152-pro-rbs2-pls.

\section{Analytical Method}

$\varepsilon$-Poly-L-lysine concentration was determined using the method described previously (Pan et al., 2017). Glucose concentration was measured using an SBA-40E biosensor analyzer (Shangdong Academy of Sciences, China) (Yin et al., 2019). The concentration of glycerol in the culture supernatant was measured using a Glycerol GK Assay Kit (Megazyme, Ireland) (Lenzen et al., 2019). Cell growth was measured by detecting the optical density (OD) of the samples at $600 \mathrm{~nm}$ in a spectrophotometer. All samples were measured three times. All the figures were plotted using GraphPad Prism 7 software (GraphPad Software, United States). Statistical analysis was performed using unpaired $t$ test or oneway ANOVA with Dunnett's multiple comparisons test or Sidak's multiple comparisons test in GraphPad Prism. 


\section{Comparison of pls Gene Expression by Quantitative Real-Time PCR}

Quantitative Real-Time PCR was used to compare the expression levels of pls gene in different $S$. albulus strains. Spores of the wild strain CICC 11022 and the genetically engineered strain Q-PL2 were collected from MS solid medium, inoculated into M3G medium, and cultured at $30^{\circ} \mathrm{C}$ with shaking for $60 \mathrm{~h}$. Samples were collected every $12 \mathrm{~h}$ for RNA isolation. Total RNA was extracted with an EasyPure ${ }^{\circledast}$ RNA Kit (Transgen, China). The cDNA was reverse transcribed using EasyScript ${ }^{\circledast}$ One-Step gDNA Removal and cDNA Synthesis SuperMix (Transgen, China), and qRT-PCR was performed using a QuantiNova SYBR Green PCR kit (Qiagen, Germany) on a LightCycler 96 instrument (Roche, Germany). The PCR conditions were as follows: $95^{\circ} \mathrm{C}$ for $5 \mathrm{~s}$ and $60^{\circ} \mathrm{C}$ for $10 \mathrm{~s}$, for 40 repeated cycles. The RNA polymerase sigma factor (hrdB) was selected as the reference gene (Xu et al., 2018). The genes and primers used for qRT-PCR are shown in Supplementary Table S2. The relative gene expression data were analyzed using the $2^{-\Delta \Delta \mathrm{Ct}}$ method, as described by Qin et al. (2015). All qRT-PCR runs were conducted with three biological and three technical replicates.

\section{RESULTS}

\section{Comparison of pls Gene Expression Levels in Wild and Genetically Engineered S. albulus}

The expression level of the pls gene at $12 \mathrm{~h}$ in S. albulus CICC 11022 was defined as 1 , and $h r d B$ was used as the internal reference gene. The relative quantitative method was used to calculate the expression levels of the pls gene at $24,36,48$, and $60 \mathrm{~h}$ in strain CICC 11022 and those of pls genes at 12, 24, 36,48 , and $60 \mathrm{~h}$ in genetically engineered strain Q-PL2. The results are shown in Figure 1A. In the wild strain CICC 11022, the expression of $p l s$ gene increased slowly with the increase of culture time, reached the maximum value at $60 \mathrm{~h}$, and had an increasing trend. The results of Yamanaka et al. (2010) showed that the pls gene is transcriptionally regulated in the mid-log and stationary phases of strain growth, which is consistent with our results of strain CICC 11022. The constitutive promoter $\mathrm{kasOp}^{*}$ was used in the genetically engineered strain Q-PL2. The expression levels of the pls gene in Q-PL2 reached the maximum at the beginning and then gradually decreased, but they were always much higher than those in CICC 11022. Figure 1B shows the relative expression folds of $p l s$ gene between Q-PL2 and CICC 11022 at different time points. These results indicate that the constitutive promoter $k a s O \mathrm{p}^{*}$ together with RBS2 can significantly improve the expression level of the pls gene, especially in the early stage of fermentation.

\section{Effect of pls Gene Expression on $\varepsilon-P L$ Production by $S$. albulus}

The $\varepsilon$-PL production ability of S. albulus CICC 11022 and the $p l s$ gene overexpressing strain Q-PL2 was compared using M3G medium. The results are shown in Figure 2. After $72 \mathrm{~h}$ of

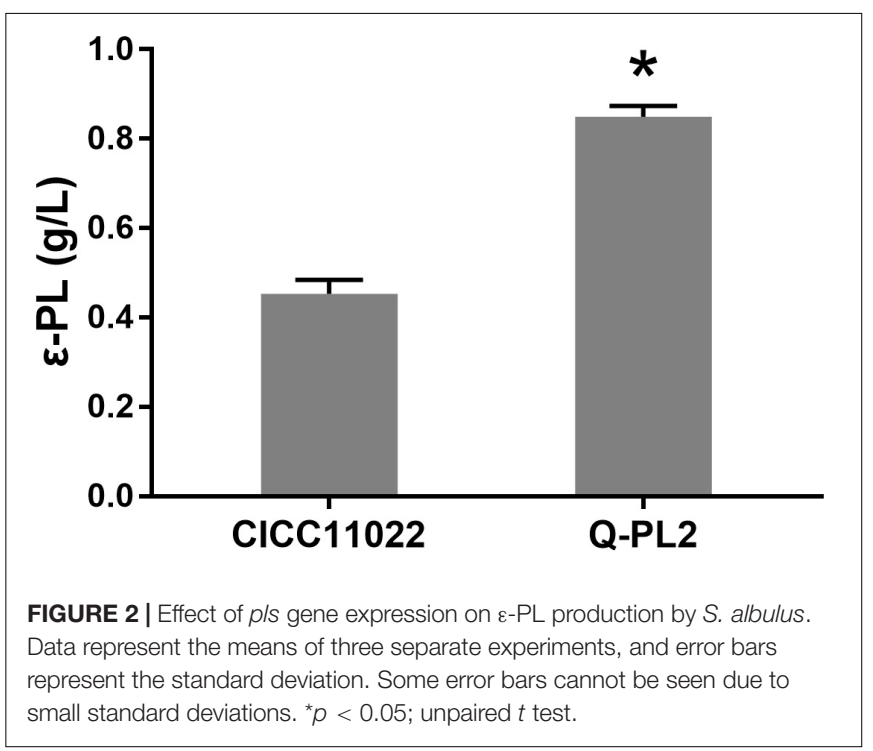

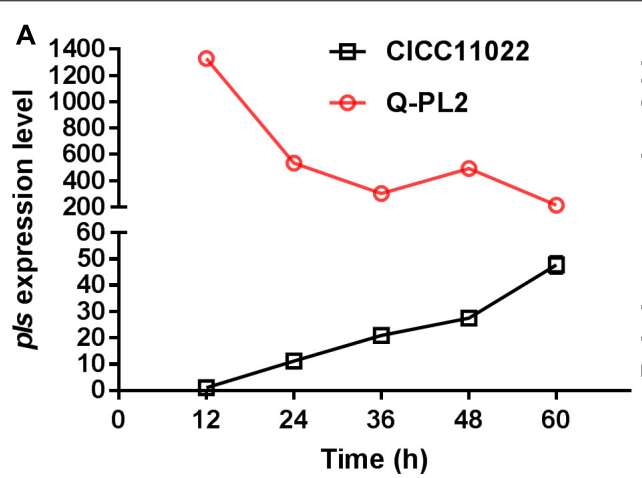

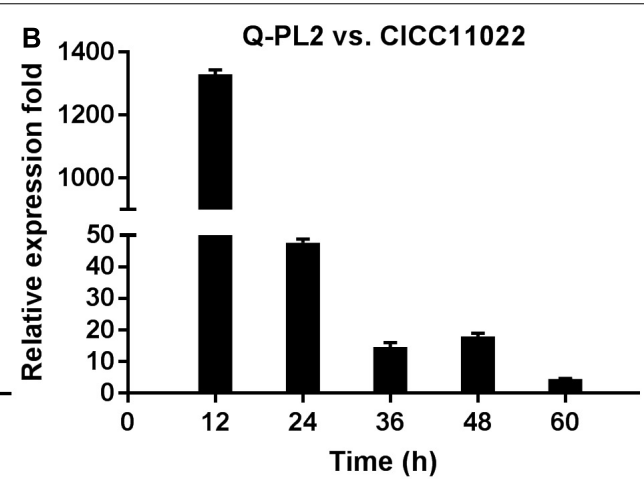

FIGURE 1 | Comparison of pls gene expression by qRT-PCR. (A) Expression level of pls gene in CICC 11022 and Q-PL2 during fermentation. (B) Relative expression fold of pls gene between Q-PL2 and CICC 11022 at different fermentation times. Data represent the means of three separate experiments, and error bars represent the standard deviation. Some error bars cannot be seen due to small standard deviations. 
fermentation, the titers of $\varepsilon$-PL produced by CICC 11022 and Q-PL2 were $0.45 \pm 0.03$ and $0.85 \pm 0.02 \mathrm{~g} / \mathrm{L}$, respectively. The latter was $88.2 \pm 8.3 \%$ higher than the former. The results confirm that $p l s$ gene overexpression can improve $\varepsilon$ $\mathrm{PL}$ production of $S$. albulus. However, the increase of $\varepsilon-\mathrm{PL}$ production did not match the increase of $p l s$ gene overexpression.

\section{Synergistic Effect of pls Gene Overexpression and Citrate on $\varepsilon-P L$ Production}

Several studies have indicated that the addition of citrate is beneficial for $\varepsilon$-PL production (Bankar and Singhal, 2011; Xia et al., 2014). Therefore, the effects of sodium citrate and other metabolic intermediates $(2 \mathrm{~g} / \mathrm{L})$ on the $\varepsilon$-PL production capacity of the pls gene overexpressing strain Q-PL2 were studied, and the results are shown in Figure 3. The titer of $\varepsilon$-PL was significantly improved by adding sodium citrate in M3G medium, while other metabolic intermediates had little effect on $\varepsilon$-PL production by strain Q-PL2.

Then, the effects of sodium citrate on the $\varepsilon$-PL production abilities of the two strains were compared, and the results are shown in Figure 4. By adding $2 \mathrm{~g} / \mathrm{L}$ sodium citrate, the $\varepsilon$-PL titers of S. albulus CICC 11022 increased from $0.45 \pm 0.03$ to $0.58 \pm 0.07 \mathrm{~g} / \mathrm{L}$, while the $\varepsilon$-PL titers of strain Q-PL2 increased from $0.85 \pm 0.02$ to $1.81 \pm 0.17 \mathrm{~g} / \mathrm{L}$. The increased ratios of $\varepsilon-\mathrm{PL}$ titers caused by sodium citrate to strain CICC 11022 and Q-PL2 were $28.5 \pm 7.0$ and $113.2 \pm 13.3 \%$, respectively. Hence, the addition of sodium citrate can synergize with the overexpression of the pls gene. With the synergistic effect of $2 \mathrm{~g} / \mathrm{L}$ sodium citrate, the titers of $\varepsilon$-PL produced by Q-PL2 were $211.2 \% \pm 17.4 \%$ higher than that produced by the wild strain.

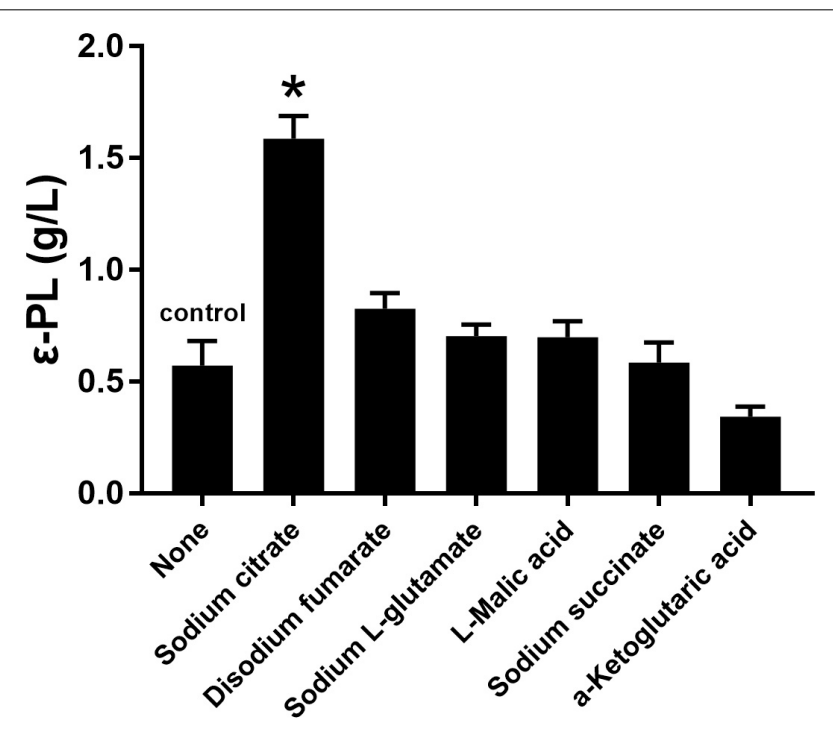

FIGURE 3 | Effects of different metabolic intermediates on $\varepsilon$-PL production by the pls gene overexpressing strain Q-PL2. Data represent the means of three separate experiments, and error bars represent the standard deviation. Some error bars cannot be seen due to small standard deviations. ${ }^{*} p<0.05$; one-way ANOVA with Dunnett's multiple comparisons test.

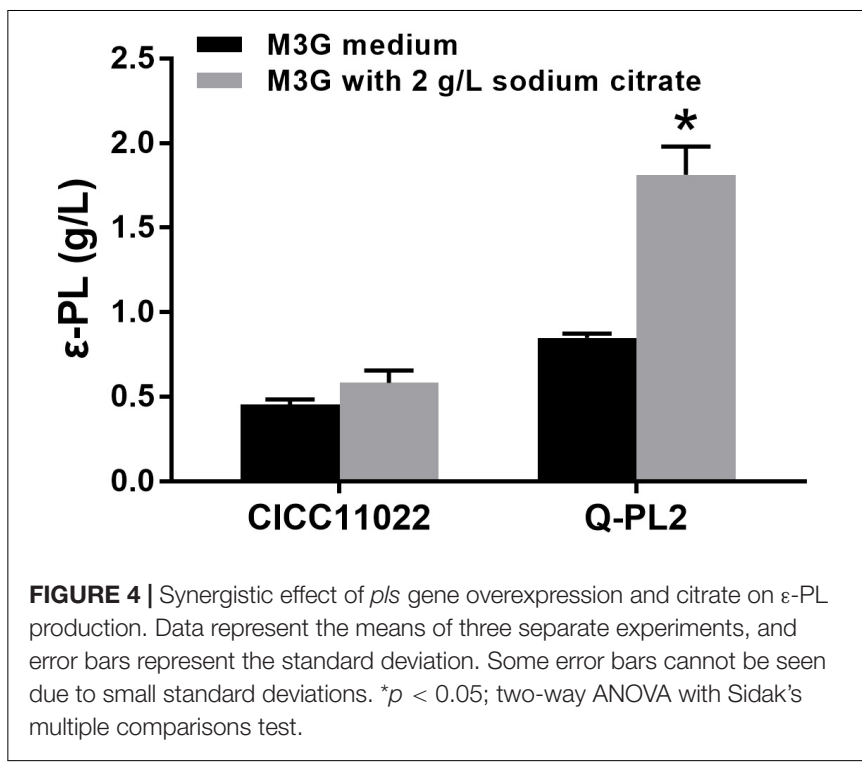

\section{Shake Flask Fermentation of $\varepsilon$-PL}

To further confirm the synergistic effect of $2 \mathrm{~g} / \mathrm{L}$ sodium citrate and $p l s$ gene overexpression, the cell growth and $\varepsilon$-PL production of the two strains at different time points were studied in shake flask fermentations. The maximum OD values of S. albulus CICC 11022 and Q-PL2 were $13.9 \pm 0.4$ and $9.5 \pm 0.2$, respectively (Figure 5A). The maximum $\varepsilon$-PL titers of $S$. albulus CICC 11022 and Q-PL2 were $0.57 \pm 0.03$ and $2.07 \pm 0.1$, respectively (Figure 5B). These results indicate that the synergistic effect of $p l s$ gene overexpression and sodium citrate can decrease cell growth and significantly increase the production of $\varepsilon-\mathrm{PL}$.

\section{Optimization of the Fermentation Conditions}

The effects of sodium citrate concentration, carbon source, seed culture time, and initial $\mathrm{pH}$ of seed medium on $\varepsilon$-PL production were studied to obtain an optimal fermentation condition for S. albulus Q-PL2. As shown in Figure 6A, the best sodium citrate concentration was $5 \mathrm{~g} / \mathrm{L}$. Zeng et al. (2017) reported that the mixed carbon source of glucose and glycerol lead to higher $\varepsilon$-PL production, and this was verified in our study. The best carbon sources were $25 \mathrm{~g} / \mathrm{L}$ glucose and $25 \mathrm{~g} / \mathrm{L}$ glycerol (Figure 6B). In addition, the best seed culture time and initial $\mathrm{pH}$ of seed medium were $48 \mathrm{~h}$ and 6.1, respectively (Figures 6C,D). These optimal fermentation conditions were applied in fed-batch fermentations.

\section{Fed-Batch Production of $\varepsilon-P L$}

To further verify the $\varepsilon$-PL production ability of the pls gene overexpressing strain Q-PL2, fed-batch fermentations were performed, and the results are shown in Figure 7. During the entire fermentation process, the cell growth of the wild strain CICC 11022 was better than that of Q-PL2, but more $\varepsilon$-PL was produced by Q-PL2. The maximum specific growth rate, product formation rate, glucose and glycerol consumption rate of S. albulus Q-PL2 were $0.56 \mathrm{~h}^{-1}, 0.63,0.70$, and $0.37 \mathrm{~g} / \mathrm{L} / \mathrm{h}$, respectively. These values of $S$. albulus CICC 11022 were, in 

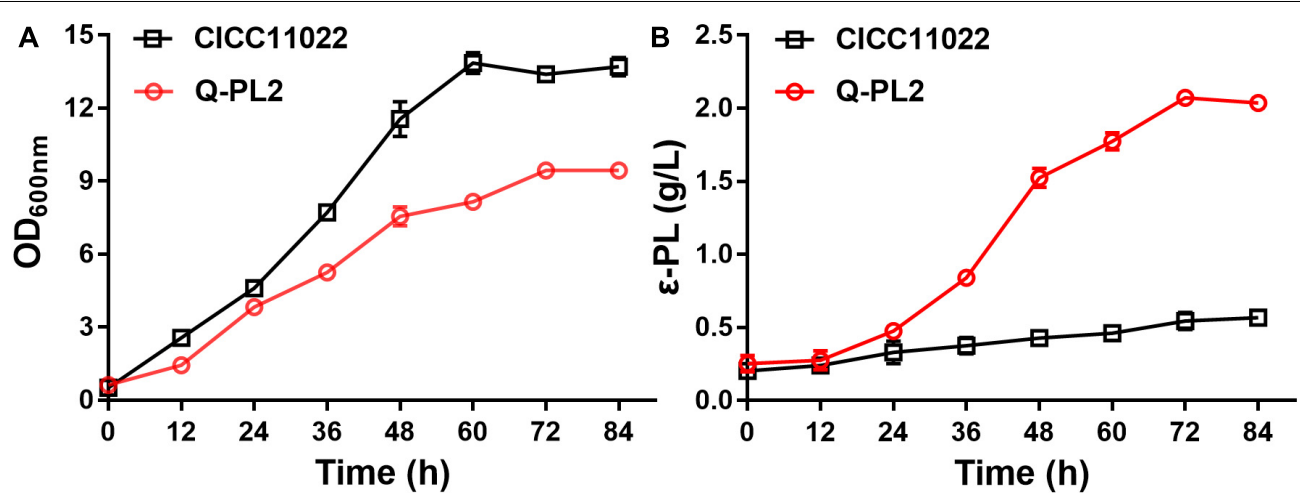

FIGURE 5 | Cell growth and $\varepsilon$-PL production of S. albulus CICC 11022 and Q-PL2 under shake flask fermentations. (A) S. albulus CICC 11022. (B) S. albulus Q-PL2. Data represent the means of three separate experiments, and error bars represent the standard deviation. Some error bars cannot be seen due to small standard deviations.
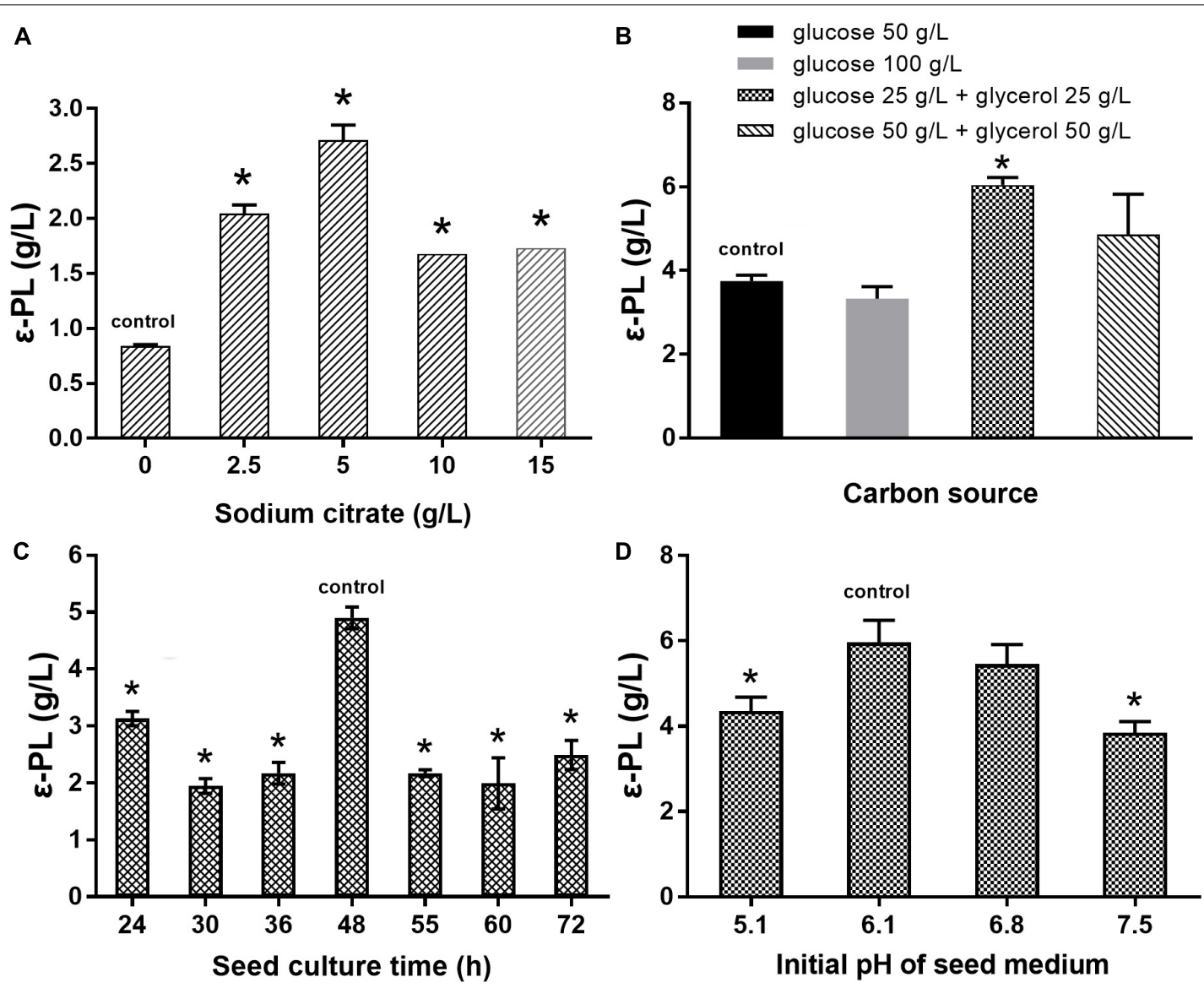

FIGURE 6 | Optimization of the fermentation conditions for $\varepsilon$-PL production by S. albulus Q-PL2. (A) Effect of sodium citrate concentration on $\varepsilon$-PL production. (B) Effects of carbon source on $\varepsilon$-PL production. (C) Effects of seed culture time on $\varepsilon-P L$ production. (D) Effects of initial pH of seed medium on $\varepsilon$-PL production. Data represent the means of three separate experiments, and error bars represent the standard deviation. Some error bars cannot be seen due to small standard deviations. ${ }^{*} p<0.05$; one-way ANOVA with Dunnett's multiple comparisons test.

turn, $0.53 \mathrm{~h}^{-1}, 0.18,0.44$, and $0.44 \mathrm{~g} / \mathrm{L} / \mathrm{h}$, respectively. The $\varepsilon$-PL yields produced by strains Q-PL2 and CICC 11022 on carbon sources were 9.1 and $3.5 \%$ (mass ratio), respectively. After $72 \mathrm{~h}$ of fermentation, $20.1 \pm 1.3 \mathrm{~g} / \mathrm{L}$ of $\varepsilon$-PL was produced with a productivity of $6.7 \pm 0.4 \mathrm{~g} / \mathrm{L} /$ day by strain Q-PL2 (Figure 7A), whereas only $6.3 \pm 0.4 \mathrm{~g} / \mathrm{L}$ of $\varepsilon$-PL was produced with a productivity of $2.1 \pm 0.1 \mathrm{~g} / \mathrm{L} /$ day by strain CICC 11022 (Figure 7B). The titer and productivity of $\varepsilon$-PL 

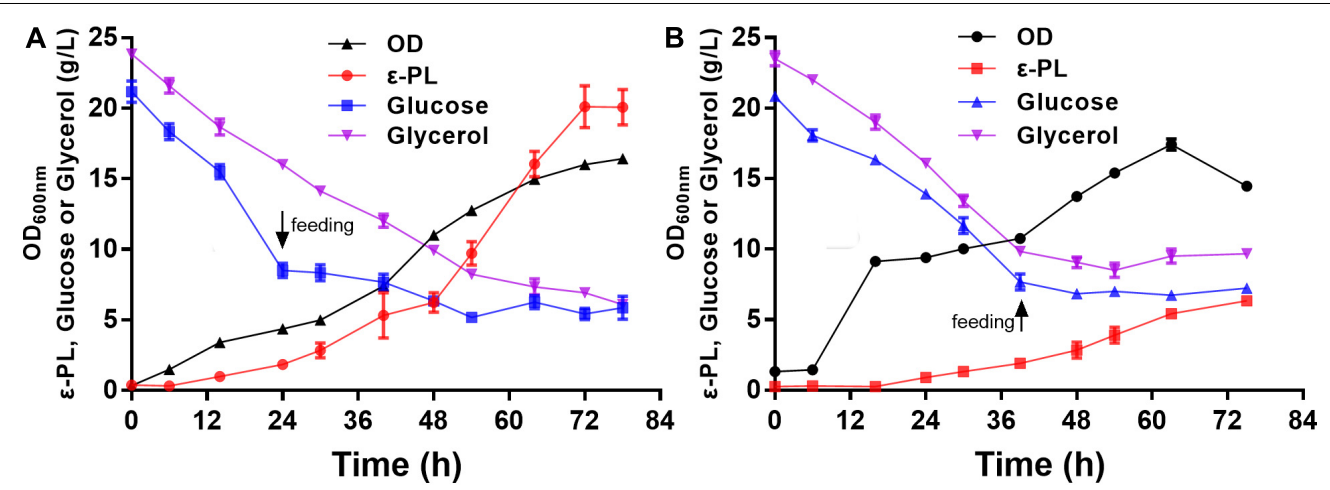

FIGURE 7 | Fed-batch fermentations of S. albulus Q-PL2 and CICC 11022. (A) S. albulus Q-PL2. (B) S. albulus CICC 11022. Data represent the means of three samples per time interval, and error bars represent the standard deviation. Some error bars cannot be seen due to small standard deviations.

produced by strain Q-PL2 was $3.2 \pm 0.3$-fold of that produced by strain CICC 11022 .

\section{DISCUSSION}

Genetic engineering is one of the important methods to increase the fermentation ability of industrial production strains. But, up to now, fewer researches on enhancing $\varepsilon$-PL biosynthesis by genetic manipulation have been reported. Among these reports, most are focused on alleviating the restriction of environmental factors on fermentation, and very few studies have focused on the key enzymes in the $\varepsilon$-PL metabolic pathway. Dissolved oxygen is one of the key factors affecting $\varepsilon$-PL production. Xu et al. (2015) integrated Vitreoscilla hemoglobin gene $(v g b)$ into the chromosome of $S$. albulus PD-1 to alleviate oxygen limitation during fermentation. Finally, the production of $\varepsilon$-PL was increased from 22.7 to $34.2 \mathrm{~g} / \mathrm{L}$ with a productivity of $4.9 \mathrm{~g} / \mathrm{L} /$ day. Gu et al. (2016) inserted the $v g b$ gene and S-adenosylmethionine synthetase gene ( $m e t K$ ) into the chromosome of S. albulus NK660 for expression, and the $\varepsilon$-PL titer increased from 0.6 to $0.76 \mathrm{~g} / \mathrm{L}$. Nitrogen source is another key factor restricting the synthesis of $\varepsilon$-PL. Each molecule of lysine contains two nitrogen atoms. Therefore, the synthesis of intracellular lysine requires more nitrogen sources. In order to solve this problem, the ammonium transporter gene $(a m t B)$ was overexpressed in S. albulus PD-1, and the production of $\varepsilon$-PL increased from 22.7 to $34.2 \mathrm{~g} / \mathrm{L}$ with a productivity of $5.1 \mathrm{~g} / \mathrm{L} /$ day (Xu et al., 2018). Aspartate kinase (Ask) is a key enzyme in the L-lysine synthetic pathway. Hamano et al. (2007) found that Ask of S. albulus NBRC14147 was partially regulated by feedback inhibition, so they constructed a mutant protein rAsk (M68V) that was completely unregulated by feedback inhibition and expressed the mutant protein rAsk in $S$. albulus CR1. As a result, the concentration of $\varepsilon$-PL increased from 12 to $15 \mathrm{~g} / \mathrm{L}$ with a productivity of $2.1 \mathrm{~g} / \mathrm{L} /$ day. In our research, the final concentration of $\varepsilon$-PL increased from 6.3 to $20.1 \mathrm{~g} / \mathrm{L}$ by overexpressing pls gene in $S$. albulus and the synergistic effect of citrate. The productivity of $\varepsilon-\mathrm{PL}$ reached $6.7 \mathrm{~g} / \mathrm{L} / \mathrm{day}$, which was much higher than other genetically engineered strains.
$\varepsilon$-PL synthase is the last enzyme in the $\varepsilon$-PL synthesis pathway. The function mechanism of this enzyme is well characterized (Yamanaka et al., 2008), but whether this enzyme is a key node that limits $\varepsilon$-PL synthesis remains unclear. Overexpression of $\mathrm{Pls}$ is expected to increase the production of $\varepsilon$-PL, but many attempts to use the constitutive promoter ermE* to overexpress Pls have failed (Yamanaka et al., 2011; Geng et al., 2014; Xu et al., 2019). In this study, an engineered strong promoter kasOp* (Wang et al., 2013) was used to achieve overexpression of the pls gene in S. albulus CICC 11022. During fermentation, the expression levels of the pls gene in the genetically engineered strain Q-PL2 were always 5 -fold higher than those in the wild strain, but the final titer of $\varepsilon$-PL was only increased by $88.9 \%$. These results indicate that the lack of intracellular L-lysine is likely to be a key factor limiting the production capacity of the pls gene overexpressing strain Q-PL2. We tried to add various metabolic intermediates to the fermentation medium and found that only the addition of citrate significantly increased the production of $\varepsilon$-PL by the pls gene overexpressing strain Q-PL2 (Figure 3). Xia et al. (2014) found that the addition of citric acid decreased the activities of pyruvate kinase, citrate synthase, and isocitrate dehydrogenase and increased the activity of aspartate aminotransferase in S. albulus PD-1. They deduced that citric acid feeding resulted in metabolic flux redistribution at the node of phosphoenolpyruvate. The metabolic pathway from phosphoenolpyruvate to tricarboxylic acid cycle was weakened and from phosphoenolpyruvate to oxaloacetate and L-aspartate was enhanced. $\varepsilon$-PL production was improved consequently because L-aspartate is the precursor of L-lysine synthesis (Xia et al., 2014). Additionally, tricarboxylic acid cycle is important for ATP generation because high levels of ATP are required for full enzymatic activity of Pls (Yamanaka et al., 2010). Therefore, we speculate that the addition of citrate can not only cause the accumulation of intracellular L-lysine, but also serves as a substrate to maintain the tricarboxylic acid cycle to produce sufficient ATP. However, even if the concentration of L-lysine and ATP is high enough, $\varepsilon$-PL cannot be produced sufficiently by the wild strain because the activity of Pls is not enough. This is probably the reason of the synergistic effect 
of pls gene overexpression and citrate on $\varepsilon$-PL production by S. albulus Q-PL2.

In summary, pls gene was overexpressed in S. albulus CICC 11022 by using a non-native promoter, and an enhanced $\varepsilon-\mathrm{PL}$ production was obtained with the synergistic effect of the gene and citrate. The results indicate that Pls is one of the rate-limiting enzymes in $\varepsilon$-PL synthesis pathway and lay a foundation for further improving the $\varepsilon$-PL production ability of $S$. albulus by metabolic engineering.

\section{DATA AVAILABILITY STATEMENT}

The datasets generated for this study are available on request to the corresponding author.

\section{AUTHOR CONTRIBUTIONS}

BY and JQ designed the research. AW and WT performed the experiments. XW, LC, and YX analyzed the data. XW and

\section{REFERENCES}

Bai, C., Zhang, Y., Zhao, X., Hu, Y., and Zhang, L. (2015). Exploiting a precise design of universal synthetic modular regulatory elements to unlock the microbial natural products in Streptomyces. Proc. Natl. Acad. Sci. U.S.A. 112, 12181-12186. doi: 10.1073/pnas.1511027112

Bankar, S. B., and Singhal, R. S. (2011). Metabolic precursors enhance the production of poly-e-lysine by Streptomyces noursei NRRL 5126. Eng. Life Sci. 11, 253-258. doi: 10.1002/elsc.201000127

Bierman, M., Logan, R., O’Brien, K., Seno, E. T., Rao, R. N., and Schoner, B. E. (1992). Plasmid cloning vectors for the conjugal transfer of DNA from Escherichia coli to Streptomyces spp. Gene 116, 43-49. doi: 10.1016/03781119(92)90627-2

Geng, W., Yang, C., Gu, Y., Liu, R., Guo, W., Wang, X., et al. (2014). Cloning of $\varepsilon$-poly-L-lysine $(\varepsilon-\mathrm{PL})$ synthetase gene from a newly isolated $\varepsilon$-PL producing Streptomyces albulus NK660 and its heterologous expression in Streptomyces lividans. Microb. Biotechnol. 7, 155-164. doi: 10.1111/1751-7915.12108

Gu, Y., Wang, X., Yang, C., Geng, W., Feng, J., Wang, Y., et al. (2016). Effects of chromosomal integration of the Vitreoscilla hemoglobin gene (vgb) and s-adenosylmethionine synthetase gene (metk) on $\varepsilon$-poly-L-lysine synthesis in Streptomyces albulus NK660. Appl. Biochem. Biotechnol. 178, 1445-1457. doi: 10.1007/s12010-015-1958-7

Hamano, Y., Nicchu, I., Shimizu, T., Onji, Y., Hiraki, J., and Takagi, H. (2007). $\varepsilon-$ Poly-L-lysine producer, Streptomyces albulus, has feedback-inhibition resistant aspartokinase. Appl. Microbiol. Biotechnol. 76, 873-882. doi: 10.1007/s00253007-1052-3

Kawai, T., Kubota, T., Hiraki, J., and Izumi, Y. (2003). Biosynthesis of $\varepsilon$-polyL-lysine in a cell-free system of Streptomyces albulus. Biochem. Biophys. Res. Commun. 311, 635-640. doi: 10.1016/j.bbrc.2003.10.033

Lenzen, C., Wynands, B., Otto, M., Bolzenius, J., Mennicken, P., Wierckx, N., et al. (2019). High-yield production of 4-hydroxybenzoate from glucose or glycerol by an engineered Pseudomonas taiwanensis VLB120. Front. Bioeng. Biotechnol. 7:130. doi: 10.3389/fbioe.2019.00130

Paget, M. S. B., Chamberlin, L., Atrih, A., Foster, S. J., and Buttner, M. J. (1999). Evidence that the extra cytoplasmic function sigma factor $\sigma e$ is required for normal cell wall structure in Streptomyces coelicolor A3(2). J. Bacteriol. 181, 204-211. doi: 10.1128/jb.181.1.204-211.1999

Pan, L., Chen, X. S., Liu, M. M., Liu, Y. J., and Mao, Z. G. (2017). Efficient production of $\varepsilon$-poly-L-lysine from glucose by two-stage fermentation using $\mathrm{pH}$ shock strategy. Process Biochem. 63, 8-15. doi: 10.1016/j.procbio.2017.08.008

Qin, J., Wang, X., Wang, L., Zhu, B., Zhang, X., Yao, Q., et al. (2015). Comparative transcriptome analysis reveals different molecular mechanisms of Bacillus
JQ wrote the manuscript. All authors read and approved the final manuscript.

\section{FUNDING}

This work was supported by the National Natural Science Foundation of China (31970086), the Natural Science Foundation of Shandong Province (ZR2017MC023), the Shandong Province Higher Educational Science and Technology Program (J18KA148), and the fund of the Beijing Engineering and Technology Research Center of Food Additives, Beijing Technology \& Business University (BTBU).

\section{SUPPLEMENTARY MATERIAL}

The Supplementary Material for this article can be found online at: https://www.frontiersin.org/articles/10.3389/fbioe. 2020.00288/full\#supplementary-material

coagulans 2-6 response to sodium lactate and calcium lactate during lactic acid production. PLoS One 10:e0124316. doi: 10.1371/journal.pone.0124316

Ren, X. D., Xu, Y. J., Zeng, X., Chen, X. S., Tang, L., and Mao, Z. G. (2015). Microparticle-enhanced production of $\varepsilon$-poly-L-lysine in fed-batch fermentation. RSC Adv. 5, 82138-82143. doi: 10.1039/c5ra14319e

Shima, S., Oshima, S., and Sakai, H. (1983). Biosynthesis of $\varepsilon$-poly-L-lysine by washed mycelium of Streptomyces albulus No-346. J. Agr. Chem. Soc. Jpn. 57, 221-226. doi: 10.1271/nogeikagaku1924.57.221

Shima, S., and Sakai, H. (1977). Polylysine produced by Streptomyces. Agric. Biol. Chem. 41, 1807-1809. doi: 10.1271/bbb1961.41.1807

Smith, M. C., Burns, R. N., Wilson, S. E., and Gregory, M. A. (1999). The complete genome sequence of the Streptomyces temperate phage $\phi \mathrm{C} 31$ : evolutionary relationships to other viruses. Nucleic Acids Res. 27, 2145-2155. doi: 10.1093/ nar/27.10.2145

Wang, W., Li, X., Wang, J., Xiang, S., Feng, X., and Yang, K. (2013). An engineered strong promoter for Streptomycetes. Appl. Environ. Microbiol. 79, 4484-4492. doi: 10.1128/aem.00985-13

Xia, J., Xu, Z., Xu, H., Feng, X., and Bo, F. (2014). The regulatory effect of citric acid on the co-production of poly( $\varepsilon$-lysine) and poly(L-diaminopropionic acid) in Streptomyces albulus PD-1. Bioprocess Biosyst. Eng. 37, 2095-2103. doi: 10.1007/s00449-014-1187-4

Xu, D., Wang, R., Xu, Z., Xu, Z., Li, S., Wang, M., et al. (2019). Discovery of a shortchain $\varepsilon$-poly-L-lysine and its highly efficient production via synthetase swap strategy. J. Agric. Food Chem. 67, 1453-1462. doi: 10.1021/acs.jafc.8b06019

Xu, D., Yao, H., Cao, C., Xu, Z., Li, S., Xu, Z., et al. (2018). Enhancement of $\varepsilon-$ poly-L-lysine production by overexpressing the ammonium transporter gene in Streptomyces albulus PD-1. Bioprocess Biosyst. Eng. 41, 1337-1345. doi: 10.1007/s00449-018-1961-9

Xu, Z., Cao, C., Sun, Z., Li, S., Xu, Z., Feng, X., et al. (2015). Construction of a genetic system for Streptomyces albulus $\mathrm{PD}-1$ and improving poly( $(\varepsilon-\mathrm{L}-$ lysine) production through expression of Vitreoscilla hemoglobin. J. Microbiol. Biotechnol. 25, 1819-1826. doi: 10.4014/jmb.1506.06084

Xu, Z., Xu, Z., Feng, X., Xu, D., Liang, J., and Xu, H. (2016). Recent advances in the biotechnological production of microbial poly( $\varepsilon$-L-lysine) and understanding of its biosynthetic mechanism. Appl. Microbiol. Biotechnol. 100, 6619-6630. doi: 10.1007/s00253-016-7677-3

Yamanaka, K., Kito, N., Imokawa, Y., Maruyama, C., Utagawa, T., and Hamano, Y. (2010). Mechanism of $\varepsilon$-poly-L-lysine production and accumulation revealed by identification and analysis of an $\varepsilon$-poly-L-lysine-degrading enzyme. Appl. Environ. Microbiol. 76, 5669-5675. doi: 10.1128/aem.00853-10

Yamanaka, K., Kito, N., Kita, A., Imokawa, Y., Maruyama, C., Utagawa, T., et al. (2011). Development of a recombinant $\varepsilon$-poly-L-lysine synthetase expression 
system to perform mutational analysis. J. Biosci. Bioeng. 111, 646-649. doi: 10.1016/j.jbiosc.2011.01.020

Yamanaka, K., Maruyama, C., Takagi, H., and Hamano, Y. (2008). ع-polyL-lysine dispersity is controlled by a highly unusual nonribosomal peptide synthetase. Nat. Chem. Biol. 4, 766-772. doi: 10.1038/ nchembio. 125

Yin, F. W., Zhu, S. Y., Guo, D. S., Ren, L. J., Ji, X. J., Huang, H., et al. (2019). Development of a strategy for the production of docosahexaenoic acid by Schizochytrium sp. from cane molasses and algae-residue. Bioresour. Technol. 271, 118-124. doi: 10.1016/j.biortech.2018. 09.114

Zeng, X., Zhao, J., Chen, X., Mao, Z., and Miao, W. (2017). Insights into the simultaneous utilization of glucose and glycerol by Streptomyces albulus M-Z18 for high $\varepsilon$-poly-L-lysine productivity. Bioprocess Biosyst. Eng. 40, 1775-1785. doi: 10.1007/s00449-017-1832-9

Conflict of Interest: The authors declare that the research was conducted in the absence of any commercial or financial relationships that could be construed as a potential conflict of interest.

Copyright $\odot 2020$ Wang, Tian, Cheng, Xu, Wang, Qin and Yu. This is an open-access article distributed under the terms of the Creative Commons Attribution License (CC BY). The use, distribution or reproduction in other forums is permitted, provided the original author(s) and the copyright owner(s) are credited and that the original publication in this journal is cited, in accordance with accepted academic practice. No use, distribution or reproduction is permitted which does not comply with these terms. 